ESJ Natural/Life/Medical Sciences

\title{
Caracteristiques Du Système D'exploitation Des Poulets Locaux Dans Deux Zones Agro-Ecologiques (Sud Forestier Et Centre Savanicole) De La Côte d'Ivoire
}

\section{Loukou N'Goran Etienne,}

Maître-Assistant, Département de Biochimie-Génétique, UFR de Sciences

Biologiques,Université Peleforo Gon-Coulibaly, Korhogo,

Côte d'Ivoire

Soro Kouhana,

Assistant, Département de Biochimie-Génétique, UFR de Sciences

Biologiques, Université Peleforo Gon-Coulibaly, Korhogo,

Soro Brahima,

Maitre-Assistant, Laboratoire de Génétiques, UFR Biosciences,

Université Félix Houphouët Boigny, Abidjan,

Côte d'Ivoire,

Rognon Xavier,

Professor in Animal genetics, UFR Génétique, Elevage et Reproduction, AgroParisTech/département SVS,

Paris, France

Kayang B. Boniface,

Senior Lecturer, Department of Animal Science, College of Agriculture \&

Consumer Sciences, University of Ghana Legon, Ghana,

Youssao Abdou Karim Issaka,

Professeur Titulaire, Département de Production et Santé Animales, Ecole

Polytechnique d'Abomey-Calavi, Université d'Abomey-Calavi,

Cotonou, Bénin

Yapi-Gnaoré Chia Valentine,

Directrice Générale du Centre International de recherche-développement sur l'élevage en zone subhumide (CIRDES),

Avenu du Gouverneur Louveau, Bobo-Dioulasso,

Burkina Faso

$\underline{\text { Doi:10.19044/esj.2021.v17n40p240 }}$

Submitted: 27 October 2021

Accepted: 17 November 2021

Published: 30 November 2021
Copyright 2021 Author(s)

Under Creative Commons BY-NC-ND

4.0 OPEN ACCESS 
Cite As:

Etienne L., Kouhana S., Brahima S., Xavier R.,Boniface K.,Issaka Y.A.K., \& Valentine Y.G.C., (2021). Caracteristiques Du Système D'exploitation Des Poulets Locaux Dans Deux Zones Agro-Ecologiques (Sud Forestier Et Centre Savanicole) De La Côte D'Ivoire. European Scientific Journal, ESJ, 17(40), 240. https://doi.org/10.19044/esj.2021.v17n40p240

\section{Résumé}

Les poulets locaux, malgré leur rôle important dans l'aviculture ivoirienne, souffrent de leur faible productivité. Pour contribuer à l'amélioration de leur productivité, une étude descriptive a été réalisée sur son élevage auprès 206 paysans natifs de deux zones agro-écologiques à l'aide d'un questionnaire. Les résultats indiquent les paysans exploitent les poulets locaux dans un système exclusivement extensif. L'aviculture villageoise est une activité dominée par les hommes $(79,60 \%)$ et la considérant comme secondaire $(97 \%)$. La plupart de ces aviculteurs sont analphabètes $(52,5 \%)$. La rusticité et la qualité organoleptique des produits constituent les principales motivations en faveur de cet élevage dans $82 \%$ cas en moyenne. Ils ont débuté l'élevage par achat (59\%) d'un petit noyau initial, par don ou par héritage (41 $\%)$ en zone de forêt et par confiage $(60 \%)$ en zone de savane. La $1{ }^{\text {ère }}$ ponte d'une poulette intervient en moyenne à 6 mois d'âge $(52,5 \%)$. Un faible effectif ( 23 poulets) composé en majorité de jeunes sujets $(55,21 \%)$, de poules $(33,19 \%)$ et de coqs $(11,40 \%)$ a été observé dans les cheptels. Le plein air intégral $(73,30 \%)$ a été le mode privilégié d'élevage en zone de savane. Les épizooties $(54,35 \%)$ et les prédateurs $(38 \%)$ ont été les premières causes de mortalité. Les produits d'élevage issus de ce système ont été utilisés en autoconsommation $(62 \%)$ en zone de forêt et vendus $(65 \%)$ en zone de savane. La levée des contraintes caractérisant le système extensif pourrait aider à l'amélioration de la productivité des poules locales.

Mots clés: Amélioration, Système Extensif, Poulet Local, Production 


\title{
Characteristics of the System of Exploitation of Local Chickens in Two Agro-Ecological Zones (South Forest and Savannah Center) of Côte d'Ivoire
}

\author{
Loukou N'Goran Etienne, \\ Maître-Assistant, Département de Biochimie-Génétique, UFR de Sciences \\ Biologiques,Université Peleforo Gon-Coulibaly, Korhogo, \\ Côte d'Ivoire \\ Soro Kouhana, \\ Assistant, Département de Biochimie-Génétique, UFR de Sciences \\ Biologiques, Université Peleforo Gon-Coulibaly, Korhogo, \\ Soro Brahima, \\ Maitre-Assistant, Laboratoire de Génétiques, UFR Biosciences, \\ Université Félix Houphouët Boigny, Abidjan, \\ Côte d'Ivoire, \\ Rognon Xavier,
}

Professor in Animal genetics, UFR Génétique, Elevage et Reproduction, AgroParisTech/département SVS,

Paris, France

Kayang B. Boniface,

Senior Lecturer, Department of Animal Science, College of Agriculture \& Consumer Sciences, University of Ghana Legon, Ghana,

\section{Youssao Abdou Karim Issaka,}

Professeur Titulaire, Département de Production et Santé Animales, Ecole

Polytechnique d'Abomey-Calavi, Université d'Abomey-Calavi,

Cotonou, Bénin

Yapi-Gnaoré Chia Valentine,

Directrice Générale du Centre International de recherche-développement sur

l'élevage en zone subhumide (CIRDES),

Avenu du Gouverneur Louveau, Bobo-Dioulasso,

Burkina Faso

\begin{abstract}
Local chickens play an important role in Ivorian poultry farming, but this animal resource suffers from its low productivity. In order to contribute to the improvement of this productivity, a descriptive study was carried out on its breeding among two hundred and six (206) farmers who were native to two agro-ecological zones using a structured questionnaire. The results indicate that these farmers are exploiting local chickens in an extensive system exclusively. Village poultry farming is an activity dominated by men (79.60\%) and considered secondary (97\%). Most of these poultry farmers are illiterate
\end{abstract}


(52.5\%) and the rusticity and organoleptic quality of the products are the main motivations for this breeding in an average of $82 \%$ cases. They began breeding by purchasing a small nucleus (59\%), by gifts or inheritance $(41 \%)$ in the Forest zone and by entrusting (60\%) in the Savannah zone. The first laying of a pullet occurs on average at 6 months of age (52.5\%) and the early pullet lays its first eggs between 4 and 6 months (13\%). A few chickens (23), the majority of which were youngsters $(55.21 \%)$, hens $(33.19 \%)$ and roosters $(11.40 \%)$, were observed in the flocks. In Savannah areas, free-range rearing (73.30\%) was the preferred method of rearing. Epizootics (54.35\%) and predators (38\%) were the main causes of mortality. The livestock products from this system were used for self-consumption (62\%) in the forest zone and sold (65\%) in the Savannah zone. Removing the constraints that characterize the extensive system could help to improve the productivity of local chickens.

Keywords: Extensive System, Local Chicken, Production

\section{Introduction}

Dans les pays ouest africains, les produits de l'aviculture familiale constituent une source permanente de revenus et servent comme moyen d'accumulation de capital (Guèye, 2003). Dans les campements ou villages isolés des grandes agglomérations, les volailles traditionnelles sont utilisées pour le troc. Un poulet est l'équivalent d'une barre de savon, d'un $\mathrm{kg}$ de sel et 5 à 8 poulets permettent de se procurer une chèvre selon Mukiibi-Muka (1992) en Ouganda. Les produits (viande et œufs) issus des poulets locaux sont très appréciés des consommateurs africains (Fotsa, 2008) qui leur octroient de meilleurs prix par rapport aux souches exotiques génétiquement améliorées (Guèye, 2002). Les produits avicoles locaux se retrouvent généralement au centre de nombreuses circonstances de la vie socio-culturelle et religieuse (Bonfoh, 1997 ; Sonaiya et al., 1989). Par ailleurs, l'aviculture traditionnelle est l'une des premières sources de protéines, d'origine animale, domestiques disponibles pour les personnes les plus vulnérables (femmes, enfants et personnes du troisième âge) et constitue un moyen de lutte contre la pauvreté dans les pays en développement (Guèye, 2004, Issa et al., 2012, Nahimana et al., 2019).

En Côte-d'Ivoire, l'aviculture familiale occupe toujours une place importante dans l'industrie avicole malgré le développement rapide du secteur avicole moderne utilisant les races exotiques. En 2013, il a été dénombré plus de 25,54 millions de volailles traditionnelles sur les 58,38 millions que comptait le cheptel avicole ivoirien (EU, 2017). Cette volaille traditionnelle est dominée par la sous-espèce Gallus gallus domesticus qui en occupe la première place. 
Malgré le rôle socioculturel et économique important joué par l'élevage traditionnel des poulets locaux, il n'existe pas de véritables programmes de valorisation encore moins de gestion de l'aviculture traditionnelle en Côte-d'Ivoire. Pourtant, la Côte d'Ivoire est déficitaire en produits carnés et laitiers (MIRAH, 2014). Son plan de relance pour le développement de l'élevage a adopté comme stratégie, l'amélioration de la production des animaux à cycle court où les volailles occupent une place de choix (MIRAH, 2013). Pour une amélioration efficiente des performances et une meilleure gestion des ressources animales locales, l'organisation des Nations unies pour l'alimentation et l'agriculture (FAO) recommande l'identification et la caractérisation des systèmes d'élevage et des animaux domestiques d'élevages (FAO, 1984). C'est dans ce contexte que la présente étude de caractérisation des systèmes d'élevage des poulets locaux a été initié dans un projet de valorisation des ressources animales avicoles des pays côtiers. Il s'agit spécifiquement de :

- caractériser les pratiques de l'élevage de la poule locale ;

- apprécier l'importance socio-économique des poulets locaux dans la vie des ménages ruraux;

- relever les contraintes s'opposant à l'amélioration de la productivité de la poule locale dans le milieu rural.

\section{Matériel et Méthodes}

\section{I.1. Milieu d'étude}

\section{I.1.1. Zone de Forêt}

Située au Sud-est de la Côte-d'Ivoire, elle est caractérisée par une végétation abondante, un climat attiéen où la pluviométrie annuelle est en moyenne de $1600 \mathrm{~mm}$, une température oscillant entre $26{ }^{\circ} \mathrm{C}$ et $32{ }^{\circ} \mathrm{C}$ et un taux d'hygrométrie dépassant $90 \%$ (FAO, 2005). Cette zone est composée de trois régions administratives dans lesquelles des circonscriptions administratives ont été ciblées (Figure 1):

$\checkmark$ la région des Lagunes est située au Sud (circonscription administrative : Alépé). Elle s'étend sur une superficie de 12 $949 \mathrm{~km}^{2}$ avec 5, 05 millions d'habitants dominés par une population jeune (INS, 2014),

$\checkmark$ la région du Sud-Comoé située à l'Est (circonscription administrative : Aboisso) faisant frontière avec le Ghana (pays côtier). Elle couvre une surface de $7619 \mathrm{~km}^{2}$ avec environ 0,6 million d'habitants et une population essentiellement jeune.

$\checkmark$ la région de l'Agnéby est à l'Ouest d'Aboisso (circonscription administrative : Agboville). Elle s'étend sur une superficie de $9093 \mathrm{~km}^{2}$ avec 0,7 million d'habitants et une population en majorité jeune (INS, 2014). 
L'économie de cette zone est basée sur l'agriculture d'exportation. De vastes étendues de cultures pérennes (cacao, café, hévéa, palmier à huile) sont exploitées.

\section{I.1.2. Zone de savane}

La zone de savane dans cette étude est située au Centre-Est de la Côted'Ivoire, une zone de transition entre la forêt et la savane. Elle est caractérisée par une végétation arborée, un climat baouléen avec une pluviométrie annuelle de $1000 \mathrm{~mm}$ en moyenne et une température variant entre $22^{\circ} \mathrm{C}$ et $37^{\circ} \mathrm{C}$. $\mathrm{La}$ zone de savane comprend deux régions administratives (Figure 1) :

$\checkmark$ la région des Lacs situé au Centre-Sud de la Côte-d'Ivoire où deux circonscriptions administratives ont été prospectées (Yamoussoukro et Didiévi). La superficie de la région des Lacs est estimée à $8663 \mathrm{~km}^{2}$ avec une population jeune et un total de 0,8 millions d'habitants (INS, 2014).

$\checkmark$ la région du Iffou, au Centre-Est (circonscription administrative : M'Bahiakro) elle couvre environ $19480 \mathrm{~km}^{2}$ avec environ 0,85 millions d'habitants et une population de jeunes.

La zone de savane a une économie basée sur les cultures de rentes (cacao, café, coton, mangue, anacarde).

\section{I.2. Choix des sites d'enquête et des propriétaires de poulets locaux}

Les sites d'enquêtes ont été choisis sur la base de critère d'éloignement entre les villages ou campements et le centre-ville. La distance minimale acceptable a été de $10 \mathrm{~km}$, elle garantit une bonne répartition des éleveurs sur les différentes zones d'enquête et une meilleure diversité à observer chez les poulets locaux.

Les propriétaires de fermes ont été choisis selon trois critères ; être disposé à se soumettre au questionnaire de l'enquête sur l'élevage de volaille, être éleveur de volaille d'au moins un an et être propriétaire d'au moins 10 têtes de poulets adultes. Ces critères de choix répondent au souci d'un bon échantillonnage afin de mieux cerner le système d'élevage de la poule locale.

\section{I.3. Réalisation de l'enquête et collecte des données}

L'enquête sur les poulets locaux a été conduite entre novembre 2006 et juillet 2007 à l'aide d'un questionnaire structuré. Cette enquête à passage unique a eu pour cible les propriétaires de volailles traditionnelles en milieu paysan, en particulier ceux qui possédaient la sous-espèce Gallus gallus domesticus. L'enquête a porté essentiellement sur les questions socioéconomiques qui concernent la situation sociale du propriétaire de volaille et sur les pratiques d'élevage telles que la conduite du troupeau, la santé animale 
et l'utilisation des produits d'élevage. Des données qualitatives et quantitatives ont été collectées auprès de 206 personnes dont 105 propriétaires de volailles en zone de forêt et 101 en zone de savane provenant de 60 villages et disposant de 4825 poulets locaux.

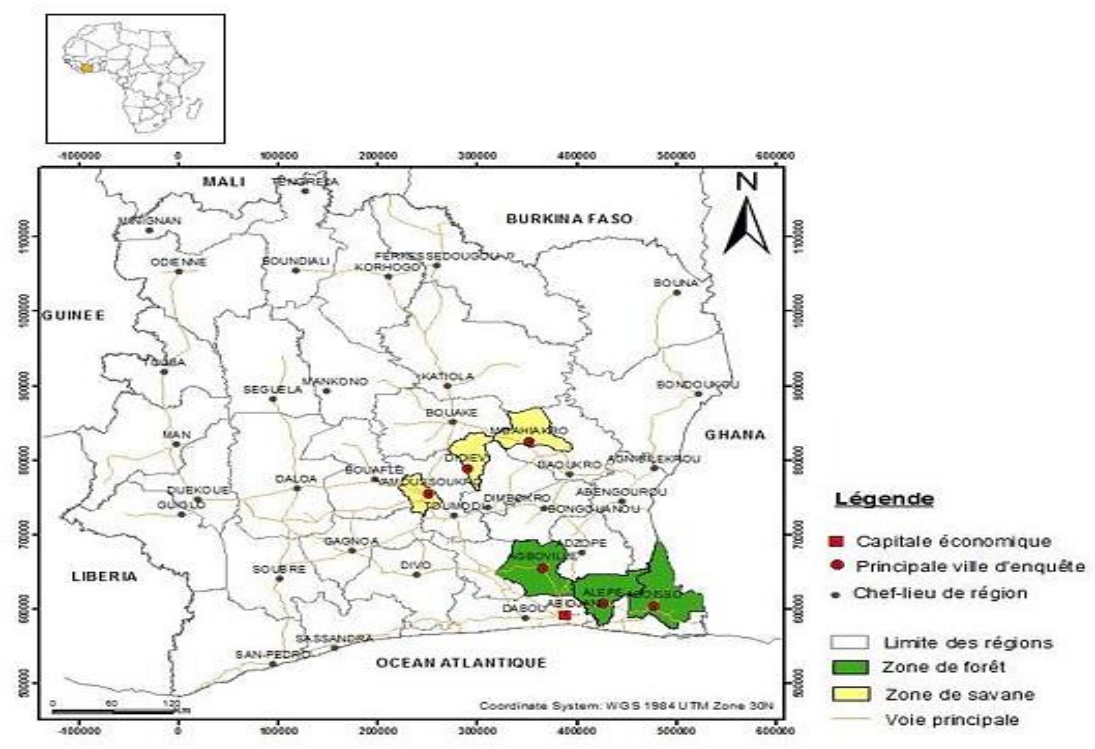

Figure 1. Départements et villes de collecte des données dans les deux zones agro-écologiques

\section{I.4. Traitement des données}

Les fréquences des variables qualitatives qui sont relatives à la situation sociale de l'éleveur, l'exploitation, la conduite du troupeau et de l'utilisation des produits d'élevage ont été calculées. Les moyennes et les écart-types des variables quantitatives (âge de l'éleveur, taille de la famille, âge de la ferme, surface de l'exploitation, taille du troupeau, paramètres de production) ont également été calculés et suivis de test $\mathrm{Z}$ ou de test Khi-deux de comparaisons des fréquences des variables entre les deux zones. La taille des différents cheptels a été obtenue par estimation selon les informations données par le propriétaire. Ces différentes analyses ont été réalisées à l'aide des logiciel XLSTAT version 2016.02.28451 et SPSS® Statistics version 20.

\section{Résultats}

\section{II.1. Statut socioculturel et économique des propriétaires de volailles}

Dans chacune des zones, l'élevage de poulets locaux est pratiqué majoritairement par les hommes, $81 \%$ et $78 \%$ en zone de forêt et de savane, respectivement (Tableau 1). Cette activité est menée par diverses personnes issues de différentes couches sociales : agriculteurs, cultivateurs, enseignants, commerçants et ouvriers agricoles. Cependant, les planteurs et les cultivateurs ont été les plus nombreux (65\%) dans les deux zones. L'élevage des poulets 
locaux est en majorité pratiqué par des adultes et des personnes du troisième âge. Ces personnes représentent $78 \%$ et $79 \%$ de l'effectif total des interviewés, respectivement en zone de forêt et en zone de savane. Ils vivent en couple dans $83 \%$ des cas et ont à $90 \%$ des enfants. Plus de la moitié des aviculteurs traditionnels n'ont pas été scolarisés (55\% de cas en zone de savane et $49 \%$ en zone de forêt). Néanmoins, sur l'ensemble des deux zones, $48 \%$ des personnes interrogées l'ont été avec $25 \%$ ayant le niveau d'étude primaire et $23 \%$, le niveau du secondaire (Tableau 1).

L'aviculture traditionnelle est considérée comme une activité secondaire par les personnes interrogées (Tableau 1) et leur activité principale dans la zone forestière est la production de cultures vivrières (riz, banane plantain, igname, taro) et/ou de cultures industrielles d'exportation (cacao, café, hévéa, palmier à huile, banane dessert). Ces deux types de productions agricoles représentent $57 \%$ de leurs activités. Par contre, en zone de savane, ces aviculteurs sont aussi producteurs de cultures vivrières (igname, manioc, arachide et légumineuses) dans $63 \%$ des cas (Tableau 1). Les autres aviculteurs sont des commerçants, des salariés agricoles, des enseignants, des infirmiers et des ouvriers agricoles.

\section{II.1.1. Types d'exploitation agricole}

En zone forestière, les personnes interrogées (40\%)ont, en plus des poulets locaux, d'autres espèces animales telles que des ruminants (36\%), des suidés et autres espèces de volailles $(4 \%)$ telles que des pintades, des canards et des dindes. En zone de savane, $43 \%$ des personnes interrogées élèvent d'autres espèces animales ; $40 \%$ de ces personnes pratiquent l'élevage de ruminants et $3 \%$ possédaient des suidés et d'autres espèces de volailles (pintades, canards et dindes).

\section{II.1.2. Motivation en faveur de l'élevage de la poule locale}

La poule locale est élevée surtout pour sa rusticité et la qualité organoleptique de ses produits (viande et œuf). Ces deux raisons ont motivé $87 \%$ des propriétaires en zone de forêt et $68 \%$ des propriétaires en zone de savane en faveur de cet élevage (Tableau 1). Certaines personnes pratiquent cette activité par simple tradition (33\%) en zone de savane.

Tableau 1. Statut socio-culturel et économique des propriétaires de poulets locaux dans les deux zones agro-écologiques

\begin{tabular}{ccccc}
\hline Variables & Modalités & Forêt & Savane & Test Z \\
\hline \multirow{2}{*}{ Sexe de l'éleveur } & Homme & $81,00 \%$ & $78,20 \%$ & NS \\
& Femme & $19,00 \%$ & $21,80 \%$ & NS \\
\cline { 2 - 4 } Tranche d'âge des & $<35$ ans & $21,90 \%$ & $20,80 \%$ & NS \\
propriétaires & 36 ans<âge<50 & $52,40 \%$ & $41,60 \%$ & NS
\end{tabular}




\begin{tabular}{|c|c|c|c|c|}
\hline \multirow{4}{*}{ Situation matrimoniale } & Age $>50$ ans & $25,70 \%$ & $37,60 \%$ & NS \\
\hline & Célibataire & $16,20 \%$ & $13,90 \%$ & NS \\
\hline & Mariage légal & $4,80 \%$ & $3,00 \%$ & NS \\
\hline & Mariage traditionnel & $79,00 \%$ & $82,20 \%$ & NS \\
\hline \multirow{5}{*}{ Formation initiale } & Primaire & $19,00 \%$ & $30,70 \%$ & NS \\
\hline & Secondaire & $30,50 \%$ & $9,90 \%$ & $* *$ \\
\hline & Supérieur & $1,90 \%$ & $3,00 \%$ & NS \\
\hline & Non scolarisé & $48,60 \%$ & $56,40 \%$ & NS \\
\hline & Agro-éleveur & $4,80 \%$ & $2,00 \%$ & NS \\
\hline \multirow[t]{3}{*}{ Formation professionnelle } & Autres & $21,90 \%$ & $24,80 \%$ & NS \\
\hline & Aucune & $73,30 \%$ & $73,30 \%$ & NS \\
\hline & Cultivateurs & $21,90 \%$ & $62,40 \%$ & $* *$ \\
\hline \multirow{4}{*}{ Activités principales } & Planteurs & $34,30 \%$ & $1,00 \%$ & $* *$ \\
\hline & Eleveurs & $3,80 \%$ & $2,00 \%$ & NS \\
\hline & Autres & $40,00 \%$ & $34,70 \%$ & NS \\
\hline & Ruminants & $36,20 \%$ & $39,60 \%$ & NS \\
\hline \multirow{3}{*}{ Autres espèces } & Porcins & $1,90 \%$ & $1,00 \%$ & NS \\
\hline & Autres & $1,90 \%$ & $2,00 \%$ & NS \\
\hline & Aucune & $60,00 \%$ & $57,40 \%$ & NS \\
\hline \multirow[t]{2}{*}{ Production agricole } & Viviers/ industriels & $94,30 \%$ & $97,00 \%$ & NS \\
\hline & Aucune & $5,70 \%$ & $3,00 \%$ & NS \\
\hline \multirow{3}{*}{ Surface exploitation } & 0 ha & $2,90 \%$ & $4,00 \%$ & NS \\
\hline & 0,25 à 1 ha & $18,10 \%$ & $26,70 \%$ & NS \\
\hline & 1,25 à 5 ha & $56,20 \%$ & $60,40 \%$ & NS \\
\hline \multirow{5}{*}{$\begin{array}{c}\text { Motivations } \\
\text { de poule locale }\end{array}$} & Plus de 5 ha & $22,90 \%$ & $8,90 \%$ & $* *$ \\
\hline & Qualité & $27,60 \%$ & $22,80 \%$ & NS \\
\hline & Rusticité & $20,00 \%$ & $19,80 \%$ & NS \\
\hline & Qualité/Rusticité & $41,00 \%$ & $24,80 \%$ & $* *$ \\
\hline & Tradition & $11,40 \%$ & $32,70 \%$ & $* *$ \\
\hline
\end{tabular}

** au seuil de 0,05, la différence entre les proportions des modlités d'une varible dans une même colonne est significative. $N S=$ Non significative au seuil de $5 \%$ 


\section{II.2. Conduite d'élevage des poulets locaux}

Les fermes visitées ont en moyenne 13 ans d'âge d'existence. La plus récente a été observée en zone de forêt et la plus ancienne en zone de savane, respectivement 1 an et 60 ans d'âge (Tableau 2).

\section{II.2.1. Acquisition du noyau initial}

Le mode d'acquisition du noyau initial est l'achat dans $59 \%$ des cas et le don ou l'héritage des volailles dans $41 \%$ des cas en zone de forêt. Par contre, en zone de savane, la réception d'une ou de deux poules confiées à un ami ou à un parent avec partage des descendants après un an d'élevage est le principal mode d'acquisition, suivi d'achat. Dans ces deux zones, la plupart des aviculteurs ont commencé leur élevage avec un petit noyau, soit une ou deux poules lorsqu'ils sont dans un village d'éleveurs de poules locales ou une ou deux poules et un coq lorsqu'ils sont isolés. La taille du troupeau initial a été généralement inférieure à 10 têtes (Tableau 2). Ils provenaient en majorité des élevages voisins, d'un parent, du village le plus proche ou d'un marché de la localité.

\section{II.2.2. Identification des poulets et taille du cheptel}

Le mode d'identification des poulets locaux le plus utilisé dans les différentes régions visitées a été la section de doigts des pattes $(51,40 \%)$. En zone de savane, en plus de section des doigts $(47,50 \%)$, d'autres personnes reconnaissent leurs oiseaux à l'aide de la couleur et des dessins du plumage dans $46 \%$ des cas (Tableau 2).

Tableau 2. Acquisition et taille du noyau d'élevage de la poule locale dans les deux zones

\begin{tabular}{|c|c|c|c|c|}
\hline Variables & Modalités & Forêt & Savane & Test $\mathrm{Z}$ \\
\hline \multirow{3}{*}{ Age de la ferme } & 1 à 5 ans & $28,60 \%$ & $40,60 \%$ & NS \\
\hline & 6 à 10 ans & $38,10 \%$ & $24,80 \%$ & NS \\
\hline & plus de 10ans & $33,30 \%$ & $34,70 \%$ & NS \\
\hline \multirow{3}{*}{$\begin{array}{l}\text { Taille du noyau } \\
\text { d'élevage }\end{array}$} & Petit & $89,50 \%$ & $96,00 \%$ & NS \\
\hline & Moyen & $7,60 \%$ & $4,00 \%$ & NS \\
\hline & Grand & $2,90 \%$ & $\mathbf{0}$ & NS \\
\hline \multirow{4}{*}{$\begin{array}{l}\text { Provenance des } \\
\text { poulets du noyau }\end{array}$} & Un seul éleveur & $51,40 \%$ & $45,50 \%$ & NS \\
\hline & Plusieurs & $18,10 \%$ & $10,90 \%$ & NS \\
\hline & Marché & $6,70 \%$ & $16,80 \%$ & NS \\
\hline & Parent & $23,80 \%$ & $26,70 \%$ & NS \\
\hline \multirow{4}{*}{$\begin{array}{c}\text { Mode } \\
\text { d'identification des } \\
\text { poulets }\end{array}$} & Plumage & $41,90 \%$ & $45,50 \%$ & NS \\
\hline & Doigts coupés & $51,40 \%$ & $47,50 \%$ & NS \\
\hline & Bagues & $1,90 \%$ & $3,00 \%$ & NS \\
\hline & Autre & $4,80 \%$ & $4,00 \%$ & NS \\
\hline
\end{tabular}

$N S$ : test Z non significatif au seuil de $5 \%$ 
Dans ces cheptels, le sex-ratio a été d'environ 1 mâle pour 3 femelles et de 23 poulets en moyenne par éleveur. Leur structuration par région, par classe d'âge et par sexe dans les deux zones d'enquêtes est consignée dans le tableau 3. Le test de comparaison de proportions relatives aux effectifs d'oiseaux des deux zones est non significatif (Tableau 3) et les sujets jeunes majoritaires ont représenté $55 \%$ de l'effectif total.

Tableau 3. Effectifs de jeunes poulets, de coqs et de poules repartis dans les deux zones agro-écologiques

\begin{tabular}{cccccc}
\hline & & Jeunes sujets & Coqs & Poules & Total \\
\hline Zone & Agboville & $59,81 \%(558)$ & $10,83 \%(101)$ & $29,37 \%(274)$ & $100 \%(933)$ \\
de forêt & Alépé & $51,65 \%(407)$ & $10,53 \%(83)$ & $37,82 \%(298)$ & $100 \%(788)$ \\
& Aboisso & $49,87 \%(389)$ & $13,72 \%(107)$ & $36,41 \%(284)$ & $100 \%(780)$ \\
Zone & Yamoussoukro & $54,55 \%(492)$ & $10,98 \%(99)$ & $34,48 \%(311)$ & $100 \%(902)$ \\
de & Didiévi & $53,83 \%(415)$ & $12,58 \%(97)$ & $33,59 \%(259)$ & $100 \%(771)$ \\
savane & M'Bahiakro & $58,83 \%(383)$ & $9,68 \%(63)$ & $31,49 \%(205)$ & $100 \%(651)$ \\
\hline Total & Forêt & $54,14 \%$ & $11,64 \%$ & $34,23 \%$ & $100 \%$ \\
& & $(1354)$ & $(291)$ & $(856)$ & $(2501)$ \\
\cline { 2 - 6 } & Savane & $55,51 \%$ & $11,14 \%$ & $33,35 \%$ & $100 \%$ \\
& & $(1290)$ & $(259)$ & $(775)$ & $(2324)$ \\
\cline { 2 - 6 } & Test Z & NS & NS & NS & NS
\end{tabular}

Au seuil de 0,05, la différence entre les proportions dans une même colonne est non significative (NS)

\section{II.3. Paramètres de production et de reproduction de la poule locale II.3.1. Choix des reproducteur(trice)s}

Dans les fermes visitées en zone de forêt, le choix du coq reproducteur est essentiellement guidé par l'aspect morphologique de l'oiseau : gros format, haut sur patte et de préférence doré ou argenté. Quant à la poule, le choix est fait sur la base du comportement : docilité, bonne couveuse, bonne protection des poussins. Par contre ce choix, en zone de savane, ces choix sont portés principalement sur l'aspect physique du coq (gros format, couleur du plumage blanche ou perdrix) et sur la docilité de la poule mère (Figure 2). 


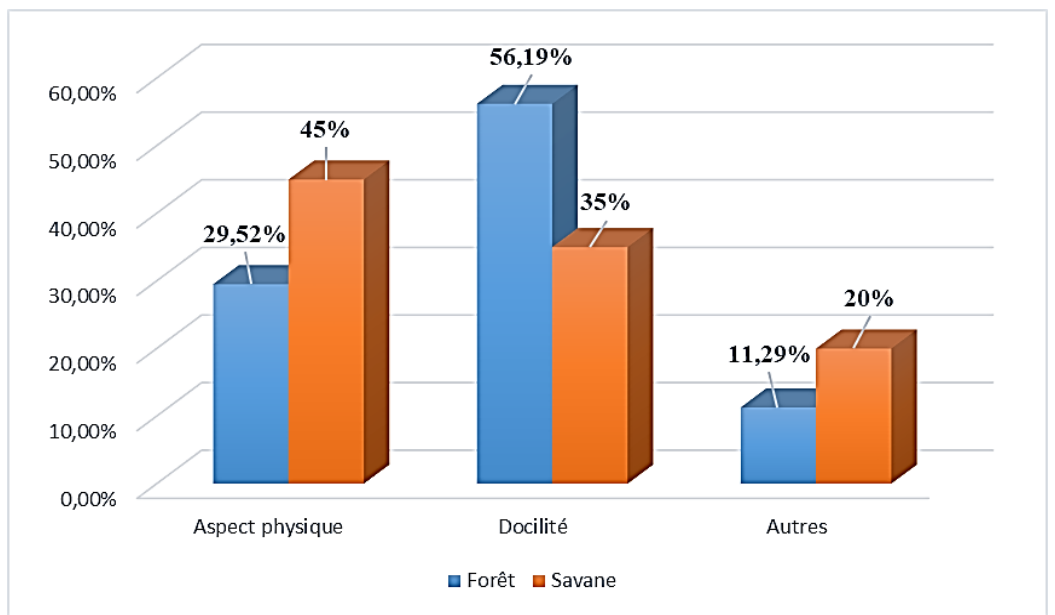

Figure 2. Fréquences des critères de choix des poules et coqs reproducteurs en zones de forêt et de savane

\section{II.3.2. Reproduction}

Dans le milieu villageois, la reproduction des poulets locaux est libre et une poule peut être fécondée par plusieurs mâles. Néanmoins, l'aviculteur peut reconnaître la mère poule de chaque oiseau de son cheptel. Après les pontes, la couvaison est réalisée naturellement sous poule aussi bien dans la zone de savane que dans la zone de forêt.

\section{II.3.3. Précocité des poulettes à la ponte}

Les poulettes locales ont effectué leur $1^{\text {ère }}$ ponte à l'âge de 6 mois en moyenne selon les personnes interrogées. Cependant, certaines poulettes rentrent en ponte entre le $4^{\mathrm{e}}$ et le $5^{\mathrm{e}}$ mois de leur vie. D'autres éleveurs ont estimé que la poulette pond ses premiers œufs à l'âge de 10 mois ou plus. Plus de la moitié des éleveurs (52\%) ont estimé que la poulette entre en ponte entre $6^{\mathrm{e}}$ et $10^{\mathrm{e}}$ mois d'âge. Les poulettes de la zone de savane semblent beaucoup plus précoces qu'en zone de forêt (Figure 3).

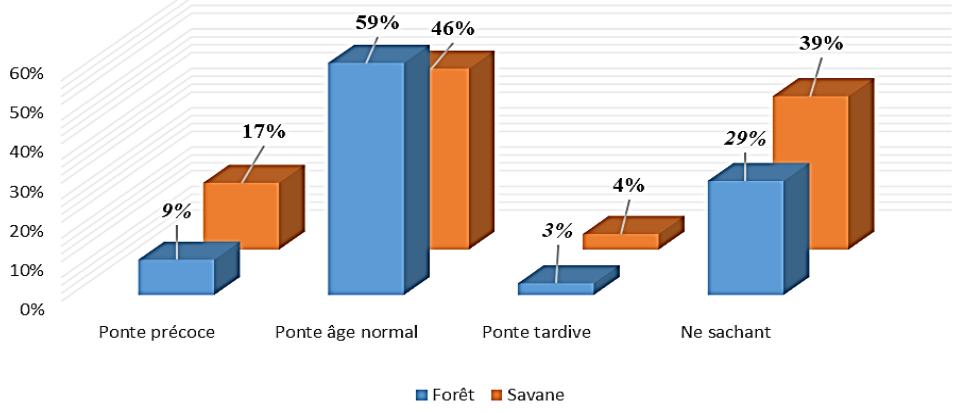

Figure 3. Fréquences de l'âge à la première ponte des poules locales dans différentes classes d'âge par les éleveurs en zones de forêt et de savane 
Ponte précoce : la lère ponte intervient chez la poulette avant l'âge de 6 mois; Ponte âge normal : la $1^{\text {ère }}$ ponte intervient entre le $6^{e}$ et le $10^{e}$ mois de vie; Ponte tardive : la $1^{\text {ère }}$ ponte intervient chez la poule après 10 mois de vie.

\section{II.3.4. Performance de ponte des poules locales}

La quantité moyenne d'œufs pondus par une poule locale est de 10 œufs par cycle de ponte. Cette quantité varie de 7 à 15 œufs en fonction de l'âge de la poule. Le cycle de ponte est estimé entre 2 et 5 par an et 4 en moyenne. La saison sèche est la période favorable à la ponte $(68 \%)$ et à l'éclosion $(80 \%)$ en zone de forêt. Quelle que soit la saison (sèche ou pluvieuse), la production d'œufs et l'éclosion s'effectuent normalement dans la zone de savane. Le taux d'éclosion pendant ces deux périodes est de $80 \%$ dans la zone de savane et $60 \%$ dans la zone de forêt.

\section{II.4. Contraintes liées à la conduite de l'élevage de la poule locale II.4.1. Habitat des poulets locaux}

Trois types d'habitat ont été observés dans les deux zones d'étude. L'abri plus parcours libre (57\%), le plein air intégral (40\%) et le bâtiment permanant $(3 \%)$ ont été observés en zone de forêt et le plein air intégral a été majoritairement observé $(73,30 \%)$ en zone de savane. Les abris sont construits à base de matériaux locaux. Ils sont faits en terre argileuse battue (banco) et couverts de pailles ou de tôles (Figure 4).
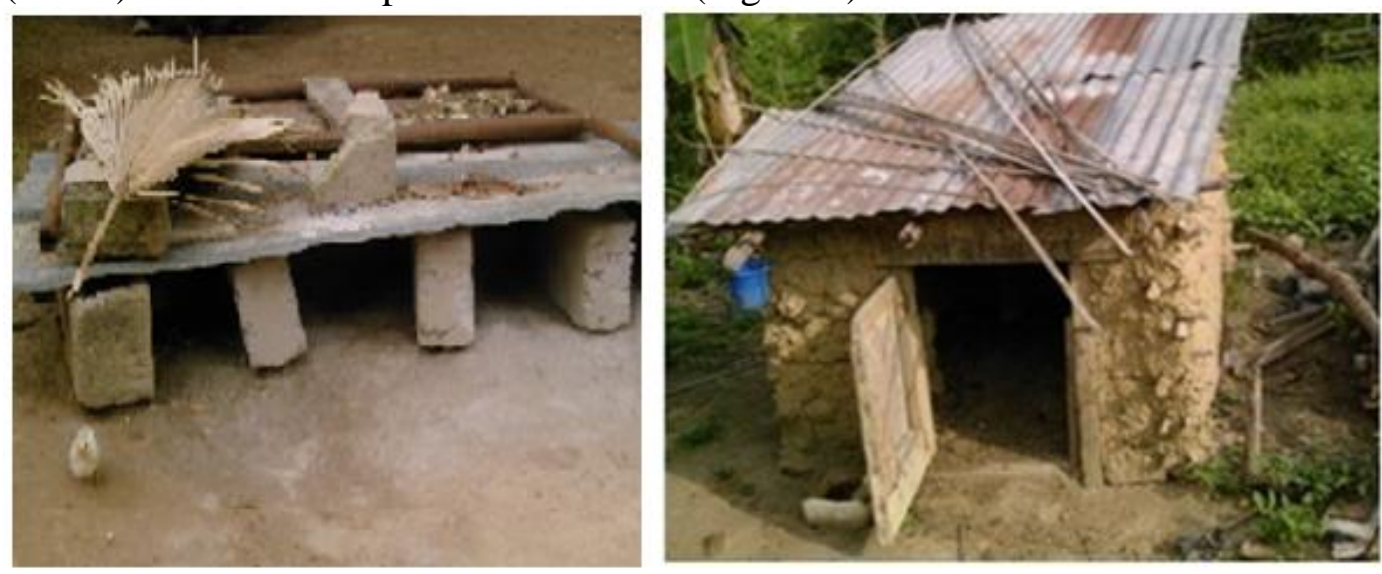

Figure 4. Abris sommaires observés des poulets locaux dans les zones d'étude

\section{II.4.2. Alimentation des poulets locaux}

La majorité $(95 \%)$ des propriétaires de volailles distribuent des compléments alimentaires à base de céréales aux poulets. D'autres $(30,5 \%)$ distribuent en plus des aliments protéiques tels que le poisson rendu en poudre et mélangé aux sons de céréales ou des termites vivants. Par contre, certains propriétaires de volailles $(9,5 \%)$ en zone de forêt n'en donnaient pas. 


\section{II.4.3. Causes de mortalité des poulets locaux}

La mortalité des oiseaux en zone de forêt a été observée surtout chez les poussins et les jeunes poulets. Les maladies aviaires ont été mises en causes par $57 \%$ des personnes interrogées dans les cas de mortalité des poulets. Ensuite les prédateurs tels que les rapaces (éperviers, corbeaux), les carnivores (civette, chats sauvages) et les reptiles (serpents) ont été cités dans $43 \%$ des cas de mortalité. En zone de savane, la mortalité a été observée à tout âge chez les oiseaux. Elle a été provoquée par des maladies aviaires dans $54 \%$ des cas de mortalité. Les prédateurs ont été à l'origine de $14 \%$ des mortalités en zone de savane (Tableau 4).

\section{II4.4. Soins administrés en cas de maladies ou de prévention aux poulets locaux}

La prévention ou le traitement des maladies aviaires est peu pratiqué par les propriétaires de volailles dans les deux zones. Seules quelques personnes utilisent des produits vétérinaires pour prévenir ou traiter les maladies aviaires ( $15 \%$ en zone de forêt et $8 \%$ en zone de savane). Plusieurs propriétaires de volailles pratiquent l'automédication (42\%) en utilisant des produits pharmaceutiques non conventionnels (de l'ampicilline communément appelée "'toupaille", du paracétamol ou de la nivaquine) pour les traitements. D'autres propriétaires utilisent des recettes médicales traditionnelles (écorces, feuilles et fruits des plantes médicinales) contre ces pathologies. En zone de savane, des éleveurs $(45,50 \%)$ de volaille n'administraient aucun traitement à leurs oiseaux en zone de savane.

Tableau 5. Conduite d'élevage des poules locales dans les deux zones agroécologiques

\begin{tabular}{|c|c|c|c|c|}
\hline Variables & Modalités & Forêt & Savanes & Test Z \\
\hline \multirow[t]{3}{*}{ Habitat des oiseaux } & $\begin{array}{l}\text { Bâtiment } \\
\text { permanant }\end{array}$ & $2,90 \%$ & $2,00 \%$ & NS \\
\hline & $\begin{array}{l}\text { Abris plus } \\
\text { parcours }\end{array}$ & $57,10 \%$ & $24,80 \%$ & $* *$ \\
\hline & Plein air intégrale & $40,00 \%$ & $73,30 \%$ & $* *$ \\
\hline \multirow{5}{*}{ Mode d'alimentation } & Céréale seule & $55,20 \%$ & $61,40 \%$ & NS \\
\hline & $\begin{array}{l}\text { Céréale + } \\
\text { compléments }\end{array}$ & $28,60 \%$ & $32,70 \%$ & NS \\
\hline & Autres aliments & $6,70 \%$ & $5,00 \%$ & NS \\
\hline & Aucune & $9,50 \%$ & $1,00 \%$ & $* *$ \\
\hline & Eau courante & $11,40 \%$ & $20,80 \%$ & NS \\
\hline \multirow{2}{*}{ Abreuvement } & Eau de puits & $67,60 \%$ & $61,40 \%$ & NS \\
\hline & Eau de marigot & $12,40 \%$ & $12,90 \%$ & NS \\
\hline \multirow{3}{*}{ Mortalité suivant l'âge } & Aucun & $8,60 \%$ & $5,00 \%$ & NS \\
\hline & Adultes & $2,90 \%$ & $8,90 \%$ & NS \\
\hline & Jeunes & $19,50 \%$ & $23,80 \%$ & $* *$ \\
\hline
\end{tabular}




\begin{tabular}{lllll} 
& Poussins & 48,60 & $41,60 \%$ & NS \\
Causes de mortalité & Pas d'âge & $29,00 \%$ & $25,70 \%$ & $* *$ \\
\cline { 2 - 5 } & Maladies & $55,20 \%$ & $53,50 \%$ & NS \\
& $\begin{array}{l}\text { Accidents (noyade } \\
\text { écrasement) }\end{array}$ & $1,90 \%$ & $13,90 \%$ & $* *$ \\
Soins apportés aux oiseaux & Prédations & $42,90 \%$ & $32,70 \%$ & $\mathrm{NS}$ \\
\cline { 2 - 5 } & Automédications & $43,80 \%$ & $40,60 \%$ & $\mathrm{NS}$ \\
& Traditionnels & $16,20 \%$ & $5,90 \%$ & $* *$ \\
& Modernes & $15,20 \%$ & $7,90 \%$ & $\mathrm{NS}$ \\
& Aucun & $24,80 \%$ & $45,50 \%$ & $\mathrm{NS}$ \\
\hline
\end{tabular}

** Au seuil de 0,05, la différence entre les proportions des modalités d'une variable dans une même colonne est significative, sinon non significative (NS)

\section{II.5. Utilisation des produits d'élevage des poulets locaux}

En zone forestière, la production de volailles est faite surtout pour l'autoconsommation (62\%). Par contre, en zone de savane, la volaille traditionnelle est produite pour la vente $(65 \%)$. Ces ventes sont périodiques ou occasionnelles. Les oiseaux les plus vendus et les plus demandés par les clients sont les mâles (79\%). Cependant, certains aviculteurs ont vendu leurs oiseaux sans tenir compte du sexe $(21 \%)$. La vente se fait en général à la ferme ou au domicile du propriétaire de volaille. Quelques rares aviculteurs (9\%) convoient leurs oiseaux au marché hebdomadaire pour la vente et d'autres ne vendent pas (11\%) leurs oiseaux. Dans la zone de forêt comme dans la zone de savane, le prix moyen de vente des poulets locaux en 2006 a été en moyenne respectivement de $2000 \mathrm{~F}$ et de $2500 \mathrm{~F}$ CFA pour la poule et le coq. Ces prix de vente ont varié de $1500 \mathrm{~F} \mathrm{CFA} \mathrm{à} 3$ 500F CFA.

\section{Discussion}

L'élevage de la poule locale est une activité secondaire pour les propriétaires de volaille en milieu rural, il est considéré comme telle par $95 \%$ des interviewés ayant des activités principales (planteurs, cultivateurs, commerçants enseignants et artisans). En zone de forêt, les propriétaires de volailles disposent de grandes surfaces d'exploitation de plus de 5 ha, par contre en zone de savane, les surfaces exploitées sont moins de 5 ha. Cette différence observée, au niveau des surfaces d'exploitation, s'explique par les activités principales qu'exercent les propriétaires de volailles surtout en zone forestière. En effet, les cultivateurs sont aussi des propriétaires de volailles et disposent de grandes surfaces pour la production agricole. L'élevage de la poule locale est dominé par les hommes $(89,60 \%)$ dans la présente étude. Ce constat pourrait s'expliquer par la gestion patriarcale de la famille en Côte d'Ivoire qui fait de l'homme est le chef de la famille, propriétaire et gestionnaire des biens. En outre, les femmes possèdent généralement des 
cheptels de petites tailles et sont éliminées par les critères de choix des personnes enquêtées (avoir au moins 10 têtes de poulets adultes). Ce qui explique la faible présence de la gente féminine dans cette étude. Un constat similaire a été fait au Sénégal (Fall et Dieng, 2017), au Niger (Amadou et al., 2010), à Dimbokro (Brou et al., 2020) où la majorité des aviculteurs ruraux sont des hommes. Par contre, dans d'autres pays tels que le Maroc, le Sénégal et le Congo, l'élevage de la poule locale est au main des femmes (Missohou et al., 2002 ; Nahimana et al., 2019, Joel et al, 2019). Plus de la moitié (52\%) des éleveurs de la poule locale sont des analphabètes, et cette proportion reflète le faible taux (55\%) d'alphabétisation de la population ivoirienne (MICS, 2016). Ces données corroborent l'idée que l'analphabétisme est une réelle entrave à l'amélioration de l'aviculture traditionnelle familiale (Samson, 2005). En effet, la plupart des éleveurs illettrés qui ont été interviewés ne font ni de la prévention, ni de traitements vétérinaires contre les maladies aviaires dans le milieu rural. Ce manque d'action aux oiseaux constitue un frein à la production de la poule locale.

Dans ces différentes zones d'étude, le propriétaire en plus d'élever la poule locale dispose dans sa ferme d'autres espèces animales telles que les ovins, les caprins, les suidés et d'autres types de volailles (pintades, canards et dindes) dans $40 \%$ des cas. Cette observation est commune à la plupart des exploitations de fermes rurales en Afrique de l'ouest où plusieurs espèces animales cohabitent. Selon des auteurs, l'aviculture familiale exploiterait plusieurs espèces animales dans une même ferme (Nahimana et al., 2019; Pindé et al., 2020) augmentant ainsi les risques de contagions entre elles.

La motivation première de l'éleveur de la poule locale dans cette étude est la qualité organoleptique des produits (œufs et viandes) d'élevage et la rusticité des poulets locaux face aux épizooties. En effet, les produits issus de l'élevage de volailles traditionnelles sont bien appréciés par de nombreux consommateurs et propriétaires de volailles. Ce résultat corrobore ceux des travaux de Kolawole (2010) au Nigéria, de Fosta (2008) au Cameroun et de Guèye (2002) au Sénégal qui affirment que ces produits sont biens appréciés et un meilleur prix est octroyé à l'achat d'une poule locale ou d'un coq traditionnel qu'à un poulet de chair (Guèye, 2002).

L'élevage de la poule locale commence par l'acquisition du noyau d'élevage. Elle se fait par dons, héritage, troc ou par achat d'une seule poule chez un éleveur voisin. Une poule peut aussi être confiée à un ami ou un parent avec partage des produits au terme d'un an de reproduction (confiage). Ces mêmes sources d'acquisition du noyau d'élevage ont été rapportées par Fotsa et al. (2007) au Cameroun. Ce système pourrait permettre aux plus démunis (femmes et jeunes) du milieu rural surtout en zone de savane de s'adonner facilement à l'élevage de la poule locale. 
Pour une meilleure surveillance et une conduite efficace d'un cheptel, l'identification des oiseaux est nécessaire. Elle a été faite de façon rudimentaire par coupure d'un doigt ou observation de couleur du plumage des poulets rendant difficile le décompte du cheptel. En effet, il est difficile à un propriétaire de connaître le nombre exact des oiseaux de son cheptel. Dans cette étude, l'effectif du cheptel a été estimé. Un cheptel de poulets locaux d'une ferme dans la présente étude compte un (1) mâle (coq) pour trois (3) femelles (poules). Ce sex-ratio obtenu est contraire aux recommandations de van Eekeren et al. (2004) avec 1 coq pour 10 poules. Il est par contre supérieur à celui observé au Mali avec 1 coq pour 14 poules (Bantiéni et Modibo, 2000). Une étude récente menée par Akoutey et al. $(20$ 18) au Bénin sur la fertilité des œufs en aviculture traditionnelle a révélée que le meilleur ratio poules-coq est de 10 pour 2 . Le rapport (1 pour 3 ) dans la présente étude donne la possibilité à l'éleveur de prélever des mâles pour l'autoconsommation, les dons, les trocs ou la commercialisation sans affecter significativement la reproduction du cheptel.

Des critères phénotypiques et comportementaux ont guidé le choix de la poule reproductrice. Pour l'éleveur, une bonne mère poule doit être docile et protéger ses poussins contre les intempéries et les prédateurs. Elle doit avoir un plumage perdrix ou fauve. Le mâle reproducteur doit être d'un grand format, haut sur pattes avec un plumage rouge doré à queue noire ou argenté. Ces critères de choix contribueraient à l'amélioration des performances de production des poulets locaux selon les éleveurs. La taille moyenne observée a été de 23 poulets par ferme et sa structuration a été de $55,21 \%(12,5)$ de jeunes sujets, $11,40 \%(2,5)$ de coqs et $33,80 \%(8)$ de poules. Tadelle (2003) a rapporté des résultats semblables ( 8,5 jeunes poulets $; 2,2$ coqs et 5,4 poules) dans 5 zones agro-écologiques différentes au Zimbabwe. Cette observation avec un effectif élevé de jeunes poulets dans cette étude est similaire aux résultats des travaux rapportés par Mammo et al. (2008) en Ethiopie où le cheptel renfermait $56 \%$ de jeunes sujets. Le faible effectif du cheptel est l'un des caractéristiques du système extensif adopté par les éleveurs dans la présente étude. Ce système est caractérisé par un effectif toujours inférieur à 100 sujets/éleveur (FAO, 2005, Samson, 2005) où les jeunes poulets sont les plus nombreux. La reproduction a été libre dans ce système où les poulets divagants s'exposent à la prédation. Les poulettes survivantes ont pondu leurs premiers œufs à 6 mois d'âge en moyenne, Ce résultat est semblable à celui des travaux d'Ayssiwede et al. (2013) qui estime l'âge à la première ponte à 25,5 semaines en Afrique subsaharienne. Des études en Côte d'Ivoire ont rapporté des premières pontes entre le $4^{\text {ème }}$ et le $5^{\text {ème }}$ mois d'âge proche des poules pondeuses exotiques estimée à 20 semaines (ITAVI, 2016). Des éleveurs estiment qu'une poulette entre en ponte à partir du $10^{\text {ème }}$ mois d'âge ; ces poules sont qualifiées de "tardive" (Ayssiwede et al., 2013). 
L'amélioration génétique de la productivité des poulets locaux pourrait se baser sur la précocité de certaines poules en milieu local. Dans les exploitations avicoles traditionnelles qui ont été visitées en 2006, le prix d'un coq se négociait entre de $2500 \mathrm{~F} \mathrm{CFA} \mathrm{à} 3500 \mathrm{~F} \mathrm{CFA}$. Quant à la poule, son prix oscillait entre $1500 \mathrm{~F} \mathrm{CFA}$ et 2 000F CFA. Au Cameroun, les prix de vente des poulets locaux sont sensiblement égaux à ceux de la Côte-d'Ivoire. Une étude semblable menée par Fotsa et al. (2007) a montré qu'un mâle est vendu à $3000 \mathrm{~F}$ CFA en moyenne, la poule est vendue à $2000 \mathrm{~F} \mathrm{CFA}$ et qu'un œuf est vendu à 100F CFA. Les prix de vente de ces produits avicoles sont intéressants pour l'éleveur qui n'investit presque pas dans ce système d'élevage extensif. Ces prix ont largement évolué au Congo où une poule se négocie entre 5 à 8 \$ (Joël et al., 2019) Un besoin pressant d'achat de médicaments ou de nourritures pourrait être résolu par la vente de 2 ou 3 coqs par l'éleveur. Une telle activité dont les produits sont comparables à une tirelire (Guèye, 2003) mérite d'être divulguée auprès des couches sociales défavorisées afin d'améliorer leurs revenus, leur alimentation et de lutter contre la pauvreté.

Différents auteurs ont montré que les races locales de poulets sont rustiques, mieux adaptées aux conditions climatiques extrêmes de divers milieux et ont une meilleure résistance à certaines pathologies aviaires (Mpupu et al., 2019; Mogesse, 2007). Cependant, plusieurs contraintes s'opposent à la valorisation de ces races locales en milieu rural. Cette étude a révélé que la petite taille du cheptel (23,43 $\pm 14,42$ têtes), le manque de suivi et de soins, la quasi-absence de logement (exposant les poulets aux aléas climatiques et environnementaux avec le ravage périodique du cheptel par les maladies saisonnières) ont été les contraintes majeures liées au système d'exploitation extensive des poulets locaux. Muchadeyi (2007), Chitate et Guta (2001) ont mis en cause ces pressions naturelles dans l'amélioration de la production et la survie de ces poulets. Les épizooties (Newcastle, Gomboro...) ont été désignées par les aviculteurs comme responsables à $57 \%$ de la mortalité de leurs oiseaux dans les deux zones agro-écologiques étudiées. Ces maladies déciment à 70-80 \% (Guèye, 1998 ; Khieu, 1998) voire même totalement le cheptel. Ces contraintes sont des facteurs de pertes de diversité génétique, car ils entraînent une érosion génétique par l'élimination de gènes portés spécifiquement par les individus qui disparaissent. La plupart de ces contraintes pourraient être levées si l'éleveur consacrait plus de temps aux soins apportés à ses oiseaux, en considérant que ces volatiles pourraient constituer une source principale de revenu et apporter à la famille de la viande de grande qualité organoleptique et nutritive. 


\section{Conclusion}

Le système d'exploitation des poulets locaux en Côte d'Ivoire est de type extensif caractérisé principalement par le faible effectif du cheptel dû aux quelques contraintes majeures qui freinent son amélioration. Ces contraintes sont : la forte mortalité due aux épizooties à certaines périodes de l'année et la forte prédation, le manque d'habitats convenables aux oiseaux pour la nuit les exposant aux intempéries et aux prédateurs la quasi absence de soins préventifs et de traitements adéquats aux poulets locaux, l'insuffisance de nourriture naturelle au cours de la divagation et le manque de complément alimentaire.

Cependant dans ce système d'exploitation extensif où la libre reproduction des volailles est la règle, le cheptel se renouvelle facilement suite à une épizootie car adapté à leur environnement.

Toute amélioration de production de la poule passerait nécessairement à la levée de ces contraintes majeures en exploitant la précocité de certaines poules et leur rusticité.

\section{References:}

1. Amadou, M.B., Idi, A., \& Benabdeljelil, K. (2010). Aviculture familiale rurale au Niger: Alimentation et performances zootechniques. RIDAF, 19(1), 5-12.

2. Akoutey A., Tosso M., Guedegbe O., Boko K., Akourki A., \& Thon A. 2018. Effets du ratio poules-coqs sur la fertilité des œufs en aviculture traditionnelle de type amélioré au Bénin.

3. Ayssiwede, S.B., Dieng, A., Houinato, M.R.B., Chrysostome, C.A.A.M., Issay, I., Hornick, J.L., and Missouhou, A. (2013). Elevage des poulets traditionnels ou indigènes au Sénégal et en Afrique Subsaharienne: état des lieux et contraintes. In Annales de Médecine Vétérinaire (Vol. 158, pp. 101-117). ULg-Université de Liège.

4. Bantiéni, T., Modibo, S. (2000). Caractéristiques des Elevages avicoles suivis par 1'APEX dans les Cercles de Kangaba et Dioïla: Définition de Rations d'appoint à tester pour l'aviculture villageoise In: Sonaiya, E.B. (ed). Issues in Family poultry Research and Development. Proceedings of an International workshop held on December 9-13, 1997 at M'Bour, Senegal, 111-131.

5. Bonfoh B. 1997. Les dominantes pathologies et les contraintes sur la productivité des poulets dans le système avicole extensif en Gambie : proposition de solution. Thèse de Doctorat de $3 \mathrm{e}$ cycle de biologie animale, $\mathrm{N}^{\circ}$ 26, Université Cheikh Anta Diop, Dakar, Sénégal.

6. Brou G.K.G., Adou C.F.D., Kouassi K.D., \& Diomandé D. 2020. Analyse technique de l'elevage du poulet traditionnel en milieu rural 
dans le Departement de Dimbokro en Cote d'Ivoire. Agronomie Africaine, 32(2), 121-134.

7. Chitate, F., Guta, M. (2001). Country Report: Zimbabwe. Alders R G and Spradbrow P B (Editors). Actes de l'atelier de planification de la SADC sur le contrôle des maladies de Newcastle des poulets villageois. Du 6 au 9 Mars 2000, Maputo, Mozambique, pp. 47-52.

8. Fall A.K., \& Dieng A. 2017. L'aviculture urbaine familiale au Sénégal: caractérisation et rôle socio-économique dans la commune de Thiès. Sciences de la vie, de la terre et agronomie, 4(2).

9. FAO. 1984. Animal genetic resource conservation by management, databanks and training. Animal Production and Health. Rome, Italy. 44 (1): 89-99.

10. FAO. 2005. Système d'information de la FAO sur l'eau et l'agriculture. http://www.fao.org/nr/water/aquastat/countries/cote divoire/indexfra. $\underline{\mathrm{stm}}$

11. Fotsa, J.C. (2008). Caractérisation des populations de poules locales (Gallus gallus) au Cameroun. Mémoire de Thèse de l'Université de Dschang, Cameroun. 301 p.

12. Fotsa, J.C., Rognon, X., Tixier-Boichard, M., Ngou, NJD., Poné, KD., Manjeli, Y. et Bordas, A. (2007). Exploitation de la poule locale en zone de forêt humide du Cameroun. Bulletin de santé et production animale en Afrique, 55 : 59-73.

13. Guèye, E.F. (1998). Poultry plays an important role in African village life. World Poultry, 14 (10) : 14-17.

14. Guèye, E.F. (2002). Employment and income generation through family poultry in low-income food-deficit countries. World's Poultry Science Journal, 58 (4): 541-557.

15. Guèye, E.F. (2003). Aviculture familiale et aviculture industrielle : Coopération au lieu de compétition. Bulletin RIDAF, 13 (2): 1-2.

16. Guèye, E.F. (2004). Evaluation d'un projet pilote d'aviculture à petite échelle financée par le Fonds International pour le développement Agricole. Etudes de terrain dans les régions de Kolda et Kaolack (Sénégal).- Rome : FIDA.- 26p

17. ITAVI. (2016). Performances techniques et coûts de production en volailles de chair, poulettes et poules pondeuses. $64 \mathrm{p}$

18. INS. (2014). Recensement Général de la Population et de l'Habitat (RGPH) 2014. https://www.ins.ci

19. Issa, Y., Mopate, L. Y., \& Missohou, A. (2012). Commercialisation et consommation de la volaille traditionnelle en Afrique subsaharienne. Journal of animal \& plant sciences, 14(3), 1985-1995.

20. Joel E.B.W.A., Monzenga J.C., Mosala F., Rutakaza N., \& José E.B. W.A. 2019. Aviculture traditionnelle dans la ville de Kisangani, 
Province de la Tshopo en République Démocratique du Congo. Revue Marocaine des Sciences Agronomiques et Vétérinaires, 7(3).

21. Khieu B. 1998. Chicken production, food security and renovative extension methodology in the SPFS Cambodia. In: Poultry as a tool in poverty eradication and promotion of gender equality. Actes d'un atelier sur la sécurité alimentaire. Du 22 au 26 Mars 1999. Tune Landboskole, Denmark

22. Kolawole, R. (2010). Frequency and Effect of Spur Gene on Metric Parameters in the Nigerian Local Chicken in a Southern Guinea Savannah Area of Nigeria (Doctoral dissertation, Master's Thesis, Department of Animal Production, Federal University of Technology Minna, Niger State, Nigeria).

23. Mammo, M., Berhan, T., \& Tadelle, D. (2008). Village chicken characteristics and their seasonal production situation in Jamma District, South Wollo, Ethiopia. Livest. Res. Rural Dev, 20.

24. MICS, (2016). Enquête à Indicateurs Multiples 2016 : La situation des femmes et des enfants en Côte d'Ivoire. Ministère du Plan et du Développement, $442 \mathrm{p}$

25. MIRAH, (2014).Plan stratégique de développement de l'élevage, de la pêche et de l'aquaculture en Côte d'Ivoire (PSDEPA 2014-2020). 2014. Tome I : Diagnostic - Stratégie de développement - Orientations stratégiques

26. MIRAH, (2015). MIRAH (2015). Filière avicole : les professionnels ivoiriens présentent l'apport d'une filière stratégique dans l'économie nationale. http://www.gouv.ci/_actualitearticle.php?d=1\&recordID $=5502 \& \mathrm{p}=5$

27. Missohou, A., Dieye, P.N., \& Talaki, E. (2002). Rural poultry production and productivity in southern Senegal. Livestock Research for Rural Development, 14(2), 2002.

28. Mogesse H.H. 2007. Phenotypic and genetic characterization of indigenous chicken populations in Northwest Ethiopia. Ph.D. Thesis. Faculty of Natural and Agricultural Sciences. University of the Free State, Bloemfontein, South Africa. 186 p.

29. Mpupu, B., Mwengi, I., Kawata, C., Kinga, D., \& Katunda, O. (2019). Revue Africaine d'Environnement et d'Agriculture. Revue Africaine d'Environnement et d'Agriculture, 2(1), 76-83.

30. Muchadeyi, F.C., Sibanda, S., Kusina, N.T., Kusina, J., Makuza, S. (2004). The village chicken production system in Rushinga District of Zimbabwe. Livestock Research for Rural Development 16 (6). http://www.lrrd.org/lrrd16/6/much16040.htm

31. Muchadeyi F.C., Sibanda S., Kusina N.T., Kusina J., Makuza S. 2005. Village chicken flock dynamics and the contribution of chickens to 
household livelihoods in a smallholder farming are in Zimbabwe. Tropical Animal Health Production 37(4): 333-334.

32. Mukiibi-Muka, G. (1992). Epidemiology of Newcastle disease in village chickens and the need to vaccinate them. Newcastle disease in village chicken. Control with thermo-stable oral vaccines. Ed. PB Spradbrow. Proceedings, (39), 155-158.

33. Nahimana, G., Ossebi, W., Missohou, A., and Ayssiwede, S.B. (2019). Analyse de l'importance socio-économique de l'aviculture familiale dans le Département de Salemata au Sénégal. International Journal of Biological and Chemical Sciences, 13(7), 3131-3143.

34. Pindé, S., Tapsoba, A. S. R., Traoré, F., Ouédraogo, R., Ba, S., Sanou, M., \& Simporé, J. (2020). Caractérisation et typologie des systèmes d'élevage de la poule locale du Burkina Faso. Journal of Animal \& Plant Sciences Vol.46 (2): 8212-8225

35. Samson K.N. (2005). Productivity and socio-cultural aspects of local poultry phenotypes in coastal kenya, M.Sc Thesis. The Royal Veterinary and Agricultural University, Copenhagen, Denmark. Sonaiya, E.B., Olori, V.E. (1989). Village chicken production in southwestern Nigeria. Dans les Actes d'un Atelier International sur le Développement de l'Aviculture Rural en Afrique. (Sonayia, E.B., Ed), 13-16 Novembre 1989, Ile-Ife, Nigeria, pp. 243-247.

36. UE, (2017). Etat des lieux des filières de production végétales et animales et revue des politiques agricoles. Côte d'Ivoire.

37. Van Eekeren, N. Maas, A. Saatkamp, H.W. and Verschuur, M. (2004). L'aviculture à petite échelle dans les zones tropicales. Agrodok $4(4 \mathrm{e}$ édn), Wageningen, Pays-Bas, 83 p.

38. Zaman M A., Sorensen P. and Howlider M R: 2006. Egg production performances of a breed and three crossbreeds under semi-scavenging system of management.

https://ir.ucc.edu.gh/xmlui/handle/123456789/4464 